Purdue University

Purdue e-Pubs

LARS Symposia

Laboratory for Applications of Remote Sensing

$1-1-1976$

\title{
Estimation of the Probability of Error without Ground Truth and Known A Priori Probabilities
}

\author{
K. A. Havens \\ T. C. Minter \\ S. G. Thadani
}

Follow this and additional works at: http://docs.lib.purdue.edu/lars_symp

Havens, K. A.; Minter, T. C.; and Thadani, S. G., "Estimation of the Probability of Error without Ground Truth and Known A Priori Probabilities" (1976). LARS Symposia. Paper 134.

http://docs.lib.purdue.edu/lars_symp/134

This document has been made available through Purdue e-Pubs, a service of the Purdue University Libraries. Please contact epubs@purdue.edu for additional information. 
Reprinted from

Symposium on

\title{
Machine Processing of Remotely Sensed Data
}

\author{
June 29 - July 1, 1976 \\ The Laboratory for Applications of \\ Remote Sensing \\ Purdue University \\ West Lafayette \\ Indiana \\ IEEE Catalog No. \\ 76CH1103-1 MPRSD \\ Copyright (C) 1976 IEEE
}

The Institute of Electrical and Electronics Engineers, Inc.

Copyright (C) 2004 IEEE. This material is provided with permission of the IEEE. Such permission of the IEEE does not in any way imply IEEE endorsement of any of the products or services of the Purdue Research Foundation/University. Internal or personal use of this material is permitted. However, permission to reprint/republish this material for advertising or promotional purposes or for creating new collective works for resale or redistribution must be obtained from the IEEE by writing to pubs-permissions@iee.org.

By choosing to view this document, you agree to all provisions of the copyright laws protecting it. 
ESTIMATION OF THE PROBABILITY OF ERROR

WITHOUT GROUND TRUTH AND KNOWN A PRIORI PROBABILITIES*

K. A. Havens, T. C. Minter and

S. G. Thadani

Lockheed Electronics Company, Inc./Aerospace Systems Division, Houston, Texas

\section{ABSTRACT}

The probability of error or, alternatively, the probability of correct classification (PCC) is an important criterion in analyzing the performance of a classifier. Labeled samples (those with ground truth) are usually employed to evaluate the performance of a classifier. Occasionally, the numbers of labeled samples are inadequate, or no labeled samples are available to evaluate a classifier's performance; for example, when crop signatures from one area from which ground truth is available are used to classify another area from which no ground truth is available. This paper reports the results of an experiment to estimate the probability of error using unlabeled test samples

(i.e., without the aid of ground truth).

\section{INTRODUCTION}

This paper presents the results of an experiment to estimate the probability of error using unlabeled samples. Two procedures, along with the test results of each, are presented. The first procedure estimates the probability of error analytically, using the posterior density function. (The analytical estimate is shown to be unbiased.) The second labels fields (for use in estimating the probability of error) simply by noting the class into which most of the field picture elements (pixels) were classified by the classifier (called the majority rule method). Empirical results from both procedures are presented, and an analysis of variance from which conclusions are drawn is performed.

*

*The material in this paper was developed under NASi Contract NAS 9-12200 and prepared for the Earth Observations Division, NASA/JSC, Houston, Texas.

\section{DESCRIPTION OF THE TWO PROCEDURES}

A. Estimating the Probability of Error Without Ground Truth

Let $\mathrm{X}$ represent an unlabeled sample which is a random, independent, $n$-dimensional measurement vector belonging to one of $\mathrm{m}$ classes. These classes are assumed to be normally distributed with a priori probabilities given by $q_{i}$ and the conaitional probability density function given by $p(x / i), i=1, \cdots, m$. Then, the overall mixture density is denoted as

$$
p(x)=\sum_{i=1}^{m} q_{i} p(x / i)
$$

The Bayes decision rule defines a Bayes region $R_{i}$ for each class $i$.

$$
\begin{aligned}
\mathrm{R}_{i} & =\left[\mathrm{x} \mid \mathrm{q}_{\mathrm{i}} \mathrm{p}(\mathrm{x} / \mathrm{i}) \geq \mathrm{q}_{\mathrm{k}} \mathrm{p}(\mathrm{x} / \mathrm{k})\right] ; \\
\mathrm{k} & =1, \cdots, \mathrm{m}
\end{aligned}
$$

Thus, the probability of error is defined as

$$
\operatorname{Pr}(E r)=\sum_{\substack{i=1 \\ i \neq j}}^{m} \sum_{\substack{j=1 \\ j}} q_{i} \operatorname{Pr}(j / i)
$$

where $\operatorname{Pr}(j / i)$ is the probability of misclassifying a sample from class $i$ into class $j$.

$$
\operatorname{Pr}(j / i)=\int_{R_{j}} p(x / i) d x
$$

Multiplying $p(j / i)$ by $\frac{p(X)}{p(X)}$ in equation

$$
\operatorname{Pr}(j / i)=\int_{R_{j}}\left[\frac{p(x / i)}{p(x)}\right][p(x) d X]
$$


Let

$$
f(i / X)=\frac{p(x / i)}{p(X)}
$$

and, if $\frac{p(x / i)}{p(x)}$ is replaced by $f(i / x)$, the result is

$$
\operatorname{Pr}(j / i)=\int_{R_{j}} f(i / X) p(x) d X
$$

From equation (7), it is shown that the probability of classifying a pixel from class $i$ into class $j$ is the "expected value" of $f(i / X)$ over the Bayes region $R_{j}$; that is,

$$
\operatorname{Pr}(j / i)=E_{R_{j}}[f(i / x)]
$$

Likewise, it can be shown that the PCC for class $i, \operatorname{Pr}(i / i)$, is

$$
\operatorname{Pr}(i / i)=E_{R_{i}}[f(i / X)]
$$

These expressions for $\operatorname{Pr}(j / i)$ and $\operatorname{Pr}(i / i)$ have two useful properties [in the following, $\operatorname{Pr}(E r)$ will represent the empirical estimate of $\operatorname{Pr}(\mathrm{Er})]$ :

\section{1. $\operatorname{Pr}(j / i)$ and $\operatorname{Pr}(i / i)$ can be estimated} using unlabeled samples.

2. An upper bound is known for the variance of $\hat{\operatorname{Pr}}(\mathrm{Er})$ in the two-class case.

To implement this analytical procedure, an assumption is made which slightly amends the computational procedure. It is assumed that the proportion of pirels classified as wheat compared to the total number of pixels is a good estimate of the a priori PCC as wheat; that is,

$$
q_{w}=\frac{\text { number of pixels classified as wheat }\left(N_{w}\right)}{\text { total number of pixels }\left(N_{T}\right)}
$$

Using equations (6) and (9),

$$
\begin{aligned}
\hat{p_{r}(w / w)} & =\frac{1}{N_{T}} \sum_{k=1}^{N} \frac{p(x / w)}{p(x)} \\
& =\frac{1}{q_{w} N_{T}} \sum_{k=1}^{N_{w}} \frac{q_{w} p(x / w)}{p(x)} \\
& =\frac{1}{N_{w}} \sum_{k=1}^{N} \frac{q_{w} p(x / w)}{p(x)}
\end{aligned}
$$

Unbiased Estimates of $p(j / i)$ and $p(i / i)$. By definition,

$$
\begin{aligned}
p(j / i) & =\int_{R_{j}} f(i / x) p(x) d x \\
& =\int_{R}\left[E(i / x) I_{R_{j}}(x)\right] p(x) d x
\end{aligned}
$$

and

$$
\begin{aligned}
p(i / i) & =\int_{R_{i}} f(i / x) p(x) d x \\
& =\int_{R}\left[f(i / x) I_{R_{i}}(x)\right] p(x) d x
\end{aligned}
$$

where

$I_{R_{j}}(X)=\left\{\begin{array}{llc}1 & \text { if } \quad X \varepsilon R_{j} \\ 0 & \text { otherwise }\end{array}\right.$

$I_{R_{i}}(X)=\left\{\begin{array}{llr}1 & \text { if } X \varepsilon R_{i} \\ 0 & \text { otherwise }\end{array}\right.$

and $f(i / X)$ and $p(X)$ are completely known functions.

If $\left\{x_{1}, \cdots, x_{N}\right\}$ denotes a sample from a population with population density function $p(x)$ and if $x_{1}{ }^{(j)}, \cdots, x_{N j}^{(j)}$ dencte observations falling in $R_{j}$, then

$$
\mathrm{N}_{1}+\cdots+\mathrm{N}_{\mathrm{m}}=\mathrm{N}
$$

where $\left[R_{1}, R_{2}, \cdots, R_{m}\right]$ is the partition and $\mathrm{N}_{j}$ denotes the number of observations falling in $R_{j}$.

$$
\begin{aligned}
\hat{p}(j / i) & =\frac{1}{N} \sum_{\alpha=1}^{N} f\left(i / x_{\alpha}\right) I_{R_{j}}\left(x_{\alpha}\right) \\
& =\frac{1}{N} \sum_{\ell=1}^{N} f\left[i / x_{\hat{\imath}}^{(j)}\right]
\end{aligned}
$$

and

$$
\begin{aligned}
\hat{p}(i / i) & =\frac{1}{N} \sum_{\alpha=1}^{N} f\left(i / x_{\alpha}\right) I_{R_{i}}\left(x_{\alpha}\right) \\
& =\frac{1}{N} \sum_{i=1}^{N} f\left[i / x_{\ell}^{(i)}\right]
\end{aligned}
$$

Thus, $\hat{p}(j / i)$ and $\hat{p}(i / i)$ are unbiased estimates of $p(j / i)$ and $p(i / i)$ since $x_{x}$ 's are 
identically distributed random variables with population density function $\mathrm{p}(\mathrm{X})$; and hence

$$
\begin{aligned}
E[\hat{p}(j / i)] & =\frac{1}{N} \sum_{\alpha=1}^{N} E\left[f\left(i / x_{\alpha}\right) I_{R_{j}}\left(x_{\alpha}\right)\right] \\
& =E\left[f(i / X) I_{R_{j}}(x)\right] \\
& =p(j / i)
\end{aligned}
$$

and

$$
\begin{aligned}
E[\hat{p}(i / i)] & =\frac{1}{\hat{N}} \sum_{\alpha=1}^{N} E\left[f\left(i / x_{\alpha}\right) I_{R_{i}}\left(x_{\alpha}\right)\right] \\
& =E\left[f(i / x) I_{R_{i}}(x)\right] \\
& =p(i / i)
\end{aligned}
$$

Variance of $\hat{\operatorname{Pr}}(\mathrm{Er})$. For clarity of presentation, an upper bound on the variance of $\operatorname{Pr}(E r)$ for a two-class case will be discussed. First, some terms will be defined. The mixture density for two classes is given by

$$
p(x)=q_{1} p(x / 1)+q_{2} p(x / 2)
$$

and

$$
p(i / x)=\frac{q_{i} p(x / i)}{p(x)}
$$

denotes the posterior probability.

When $X$ is classified according to the Bayes decision rule, the conditional probability of error is

$$
r(x)=\min [p(1 / x), p(2 / x]
$$

When the expectation of $r(X)$ is taken with respect to the random vector $\mathrm{X}$, the Bayes error is given by

$$
\operatorname{Pr}(E r)=E[r(X)]
$$

and the Bayes error can be estimated by the sample mean of $r\left(X_{i}\right)$ for $N_{t}$ test samples as

$$
\hat{\operatorname{Pr}}(\mathrm{Er})=\frac{1}{\mathrm{~N}_{\mathrm{T}}} \sum_{i=1}^{\mathrm{N}_{\mathrm{T}}} r\left(\mathrm{X}_{i}\right)
$$

This, the variance of $\hat{\operatorname{Pr}}(\mathrm{Er})$ is

$$
\operatorname{var}[\hat{\mathrm{P}} r(\operatorname{Er})]=\frac{\operatorname{var}[r(\mathrm{X})]}{\mathrm{N}_{\mathrm{T}}}
$$

Since

$$
0 \leq r(X) \leq \frac{1}{2}
$$

then

$$
r^{2}(x) \leq \frac{1}{2} r(x)
$$

and

$$
E\left[r^{2}(X)\right] \leq \frac{1}{2} E[r(X)]=\frac{1}{2} \operatorname{Pr}(E r)
$$

Therefore, a bound on $\operatorname{var}[r(x)]$ is

$$
\begin{aligned}
\operatorname{var}[r(X)] & =E\left[r^{2}(X)\right]-\operatorname{Pr}^{2}(E r) \\
& \leq \frac{1}{2} \operatorname{Pr}(E r)-\operatorname{Pr}^{2}(E r) \\
& =\operatorname{Pr}(E r)[1-\operatorname{Pr}(E r)]-\frac{1}{2} \operatorname{Pr}(E r)
\end{aligned}
$$

Thus,

$$
\begin{aligned}
\operatorname{var}[\hat{\operatorname{Pr}}(\mathrm{Er})] \leq & \frac{1}{\mathrm{~N}_{\mathrm{T}}}\{\operatorname{Pr}(\mathrm{Er})[1-\operatorname{Pr}(\mathrm{Er})] \\
& \left.-\frac{1}{2} \operatorname{Pr}(\mathrm{Er})\right\}
\end{aligned}
$$

On the other hand, if class identification were available for the $N_{m}$ test samples and an empirical error count were made, the error count would also give an unbiased estimate $\hat{P r}^{\prime}$ (Er) of the Bayes error. The variance of this estimate is known to be 2

$$
\operatorname{var}\left[\hat{\operatorname{Pr}}{ }^{\wedge}(E r)\right]=\frac{\operatorname{Pr}(E r)[1-\operatorname{Pr}(E r)]}{N_{T}}
$$

Thus, a reduction in variance can be achieved by using $\operatorname{Pr}(E r)$.

\section{B. Majority Rule Field Identification Procedure}

The second estimate of the probability of error will be computed after the classification procedure is completed. The test fields are labeled wheat or nonwheat to agree with the classification of the majority of pixels in the particular field. This estimate will be called the majority rule field identification procedure.

Let $\mathrm{N}_{i}$ be the total number of pixels in field $i$, where $i=1, \cdots, m$, and let $N_{w}$ be the total number of pixels in all of $W$ those fields identified as wheat fields from the majority pixel count; $\mathrm{N}_{\mathrm{Cw}_{j}}$ will be the total number of pixels classified as wheat in field $i$. The probability of correctly classifying wheat will be estimated by

$$
\begin{aligned}
\hat{\operatorname{Pr}}(w / w)= & \frac{\mathrm{N}_{1}}{\mathrm{~N}_{\mathrm{w}}} \operatorname{Pr}_{1}(w / w)+\frac{\mathrm{N}_{2}}{\mathrm{~N}_{\mathrm{w}}} \operatorname{Pr}_{2}(w / w) \\
& +\cdots+\frac{\mathrm{N}_{\mathrm{m}}}{\mathrm{N}_{\mathrm{w}}} \operatorname{Pr}_{\mathrm{m}}(\mathrm{w} / \mathrm{w})
\end{aligned}
$$


where the probability of correctly classifying wheat in field $i$ is given by

$$
\hat{\operatorname{Pr}} r_{i}(w / w)=\frac{{ }^{N} w_{i}}{N_{i}}
$$

Thus,

$$
\operatorname{Pr}(w / w)=\frac{1}{N_{w}} \sum_{i=1}^{m} N_{c w_{i}}
$$

\section{DESCRIPTION OF THE EXPERIMENT}

The data used in this experiment are multispectral scanner (MSS) data taken from the Earth Resources Technology Satellite (ERTS-1). [The ERTS-1 was renamed the first Land Satellite (Landsat-1) in January 1975.] The intensive test sites (ITS's) are 9 - by 11-kilometer (5- by 6-nm) tracts in Morton and Finney Counties, Kansas, for which ground truth is available.

The test fields were chosen by randomly selecting 100 fields for each county from the ground truth information. Thirty training fields were chosen for each county, first by clustering the ITS into 30 clusters and then by selecting a representative number (10 wheat, 20 nonwheat) of fields of homogeneous clusters.

The classification procedures were applied to the test sites, and a paired ttest was used in evaluating the performance of the analytical and pixel-count procedures. In both cases, this test used the difference between the estimated PCC and the true PCC (TPCC). Additionally, analysis-of-variance tables were done for the PCC, the estimated probability of classification as wheat* (PCW), and the estimated probability of classification as "other" (PCO), as calculated from the analytical procedure.

\section{RESULTS OF THE EXPERIMENT}

By means of the analytical procedure, the random test fields in each county were classified using various combinations of ERTS- 1 passes. The PCC was approximated by systematic samplings of the entire test area using the analytic estimate:

$$
P C C=q_{w} p(w / w)+q_{O} p(0 / 0)
$$

To compare estimated PCC with the TPCC, a paired t-test was performed and is presented

*In this paper, PCW and PCO will denote the probability of correctly classifying wheat and "other," respectively, as calculated by either the analytical procedure or the majority rule procedure. in table 1. Each entry in the column "mean PCC" represents the average of 30 estimates of PCC, each arising from a separate systematic sample from the same classification run. The rows of this table refer to the biological phase or phases and site used in that classification run. The t-test indicates that the PCC is a biased estimator. Upon further examination, the estimates of * PCC were found to be 6.0 percentage points too high. The tolerance $\left( \pm t .05^{\sigma}\right)$ of the

bias was calculated to the \pm 1.5 percentage points.

Figure 1 compares the computed probability with the TPCC and shows the theoretical line on which the points should be found. Again, the PCC is biased toward a slightly higher estimate.

All approximations were tested further by an analysis of variance on the differences between the estimates and true values.

For the PCW, no significant differences were found among the sites or phases in the performance of the estimate. However, the mean F-value was significant. The bias was found to be 11 percentage points higher than the true value. The tolerance $\left( \pm t .05^{\sigma}\right)$ of the bias was calculated to be \pm 6.6 percentage points. This supports the conclusion that the PCW, on the average and over all phases, overestimates the true values.

The PCO was found to be unbiased over sites, phases, or means.

For the PCC, significant differences were encountered in the means and sites. The average overestimation for Morton County was found to be 8.0 percentage points and for Finney County 4.0 percentage points. These corroborate the fact that the bias was 6.0 percentage points high over both sites. (It should be noted that only two sites were used, and the inferences made here must be confined to these particular sites.) Thus, the PCC is a consistent overestimator of the true value.

The results of using the pixel-count procedure to compare the estimated with the true values of the PCW and PCO are given in table 2. The statistical analysis of the pixel-count procedure includes the student $t$-statistic for the PCW and PCO for all test combinations of passes. Most of the statistics indicated significance at the 5 -percent level with no apparent dependence on the passes used. At this point the results from table 2 were graphed. From figure 2, the pixel-count estimate of the PCO seems to be insensitive to the TPCO. However, the range of true values is not wide enough to draw definite conclusions. 
From figure 3, the estimate of the PCW becomes more inaccurate as the TPCW takes values less than 90 percent.

\section{REFERENCES}

1. Fukunaga, K.; and Kessel1, D. L. "Nonparametric Bayes Error Estimation Using Unclassified Samples." IEEE Transactions on Information Theory, Vol. IT-19, July 1973.

2. Fukunaga, K. Introduction to statistical Pattern Recognition. Academic Press (New York), 1972, p. 146.

Table 1. Computational Results of Analytical Procedure with Paired $\mathrm{T}$-Test on PCC

\begin{tabular}{|c|l|c|c|c|}
\hline Kansas ITS & $\begin{array}{c}\text { Biological } \\
\text { phase }\end{array}$ & $\begin{array}{c}\text { Mean } \\
\text { PCC }\end{array}$ & TPCC & $\Delta$ \\
\hline Morton County & 1 & .864786 & .751103 & .113683 \\
& 2 & .939788 & .890700 & .049088 \\
& 3 & .759938 & .684564 & .075374 \\
& 4 & .885723 & .819559 & .066164 \\
& 1,2 & .974095 & .897987 & .076108 \\
& $1,2,3$ & .972914 & .889166 & .083748 \\
& $1,2,3,4$ & .985543 & .885331 & .100212 \\
\hline Finney County & 1 & .801935 & .787341 & .014594 \\
& 2 & .914815 & .887302 & .027513 \\
& 3 & .968512 & .933616 & .034896 \\
& 4 & .946467 & .901196 & .045271 \\
& 1,2 & .954650 & .902354 & .052296 \\
& $1,2,3$ & .988710 & .940178 & .048532 \\
& $1,2,3,4$ & .991599 & .934002 & .057597 \\
\hline
\end{tabular}

Mean $=0.060363$

Variance $=0.0007596$

Standard deviation $=0.007366$

T-statistic $=8.1948$

Bias $=6.04$

Tolerance of bias $= \pm 1.51$
Table 2. Computational Results for Pixel-Count Procedure

\begin{tabular}{|l|c|c|c|c|c|c|}
\hline \multicolumn{1}{|c|}{ Pass } & PCC & TPCC & PCW & TPCW & PCO & TPCO \\
\hline 1 & .864786 & .751103 & .745967 & .385343 & .889899 & .926767 \\
2 & .939788 & .890700 & .951765 & .888889 & .933004 & .891570 \\
3 & .759938 & .684564 & .663261 & .303191 & .782292 & .867726 \\
4 & .885723 & .819559 & .900269 & .759462 & .831856 & .848425 \\
1,2 & .974095 & .897987 & .977242 & .930260 & .972150 & .882865 \\
$1,2,3$ & .972914 & .889166 & .975404 & .937943 & .971287 & .865739 \\
$1,2,3,4$ & .985543 & .885331 & .983918 & .968085 & .986713 & .845586 \\
\hline & \multicolumn{7}{|c|}{ Morton County } \\
\hline 1 & .801935 & .787341 & .676597 & .544186 & .840346 & .867934 \\
2 & .914815 & .887302 & .826309 & .730232 & .940840 & .939363 \\
3 & .968512 & .933616 & .942830 & .888372 & .977528 & .948612 \\
4 & .946467 & .901196 & .932542 & .934884 & .952384 & .890031 \\
1,2 & .954650 & .902354 & .904808 & .756589 & .969130 & .950668 \\
$1,2,3$ & .988710 & .940178 & .977139 & .889922 & .992654 & .956834 \\
$1,2,3,4$ & .991599 & .934002 & .988017 & .936434 & .993025 & .933196 \\
\hline
\end{tabular}

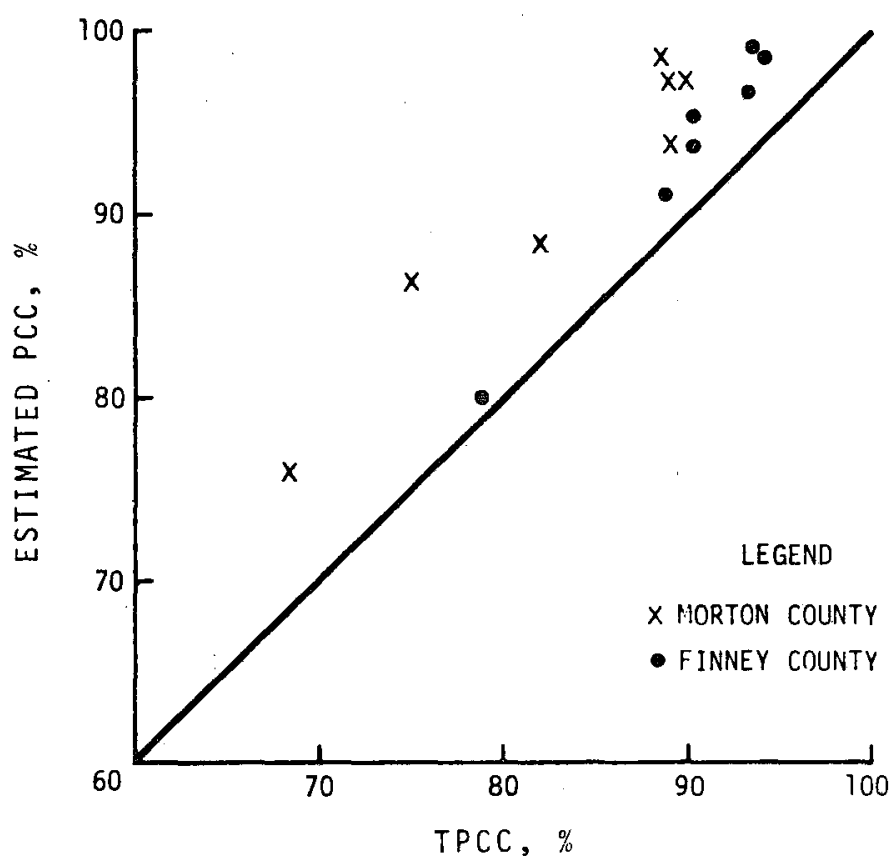

Figure 1. Comparison of Estimated Versus True Probability of Correct Classification for Analytical Procedure. 


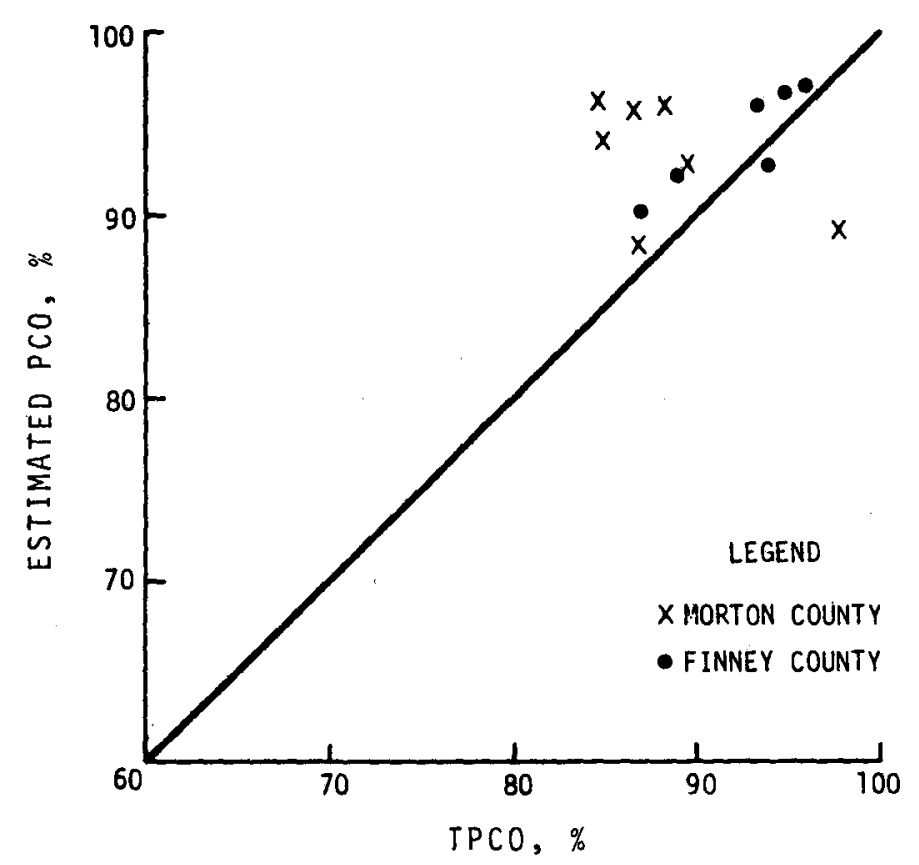

Figure 2. Comparison of the Pixel-Count Estimate Versus the True Probability of Classification as "Other."

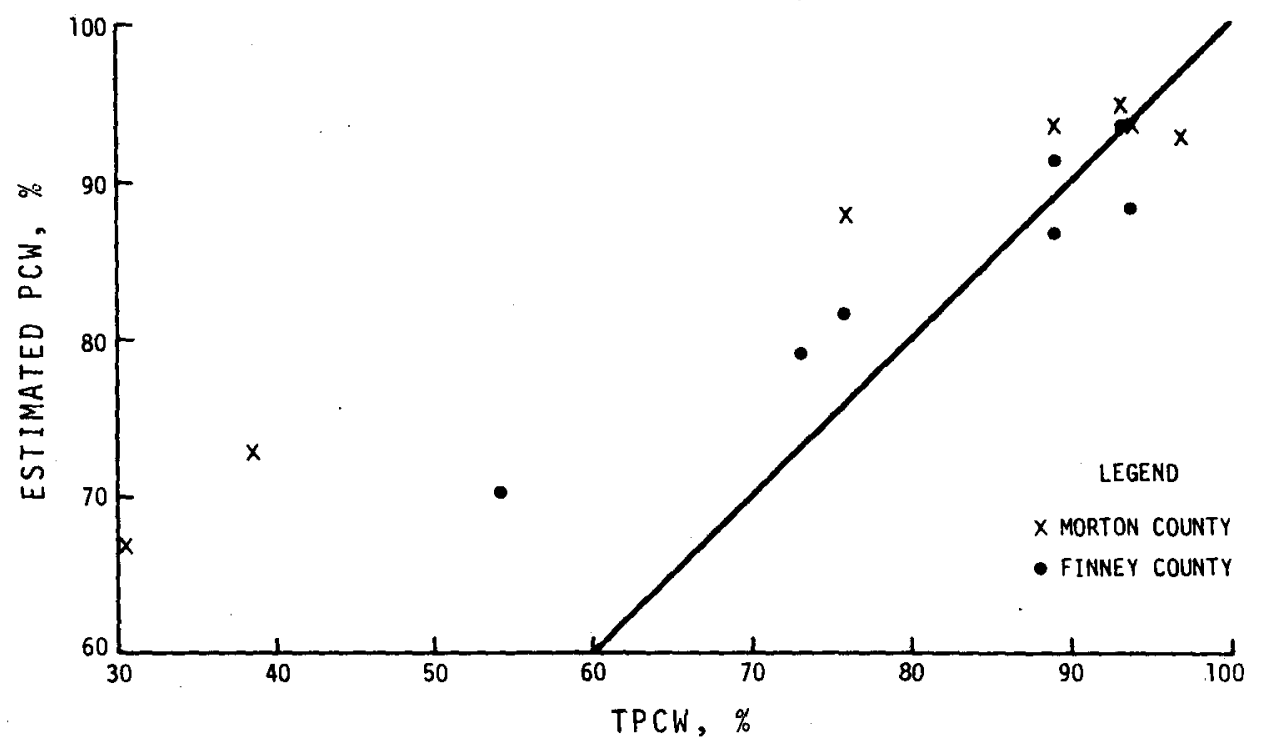

Figure 3. Comparison of the Pixel-Count Estimate Versus the True Probability of Classification as Wheat. 